\title{
含有吲哚并咔唑基团的热激发延迟荧光双极性主体材料的
}

\author{
设计、合成及应用

\begin{tabular}{|c|c|c|c|c|}
\hline 叶中华 ${ }^{\dagger}, a$ & 杨佳丽 ${ }^{\dagger}, a$ & 凌志天 ${ }^{a}$ & 赵 艺 ${ }^{a}$ & 陈 \\
\hline & 郑燕琼 $b$ & 魏＼cjkstart斌*,b & 施 鹰*,a & \\
\hline
\end{tabular}

\begin{abstract}
摘要 设计合成了一种含吲哚并咔唑基团的有机电致发光主体材料 10-苯基-10-(4-(7-苯基吲[2,3- $-b]$ 咔唑-5(7H)-基)苯基) 萝-9-(10H)-酮(DphAn-5PhIdCz). 通过核磁氢谱对其结构进行了表征, 测试了它的紫外-可见吸收波长、苂光发射波长、 荧光量子产率和瞬态荧光寿命等光物理性能, 该材料具有双极性和热延迟苂光特性. 通过将 DphAn-5PhIdCz 作为绿色 发光体(ppy) $)_{2}$ Iracac 的主体, 制备了高效低滚降的磷光有机电致发光器件(PhOLED), 其最大发光效率达到 $56.12 \mathrm{~cd} \cdot \mathrm{A}^{-1}$, 外量子效率 $15.70 \%$, 最大功率效率 $71.3 \mathrm{~lm} \cdot \mathrm{W}^{-1}$. 这些数据表明 DphAn-5PhIdCz 作为 PhOLED 主体材料在有机发光二 极管(OLED)显示和照明具有较大的潜在应用价值.
\end{abstract}

关键词＼cjkstart吲哚并咔唑; 有机发光二极管; 主体材料; 双极性; 热激发延迟

\section{Synthesis of Host Material Containing Indolocarbazole Group Featuring Bipolar and Thermally Activated Delayed Fluorecence and Its Application}

\author{
Ye, Zhonghua ${ }^{\dagger, a} \quad$ Yang, Jiali ${ }^{\dagger}, a$ \\ Ling, Zhitian ${ }^{a}$ \\ Zhao, $\mathrm{Yi}^{a}$ \\ Chen, $\mathrm{Guo}^{b}$ \\ Zheng, Yanqiong ${ }^{b}$ \\ Wei, Bin ${ }^{*, b}$ \\ Shi, Ying ${ }^{*, a}$ \\ ( ${ }^{a}$ School of Materials Science and Engineering, Shanghai University, Shanghai 200072) \\ ( ${ }^{b}$ Key Laboratory of Advanced Display and System Applications, Ministry of Education, Shanghai University, Shanghai
} 200072)

\begin{abstract}
The novel host material containing indolocarbazole group, 10-phenyl-10-(4-(7-phenylindolo[2,3-b]carbazol-5(7H)yl)phenyl)anthracen- $9(10 H)$-one (DphAn-5PhIdCz), was designed and synthesized. The structures were characterized by NMR and their photophysical properties such as ultraviolet-visible absorption wavelength, fluorescence emission wavelength, fluorescence quantum yield, and transient fluorescence lifetime were measured. The DphAn-5 PhIdCz was found to exhibit the characteritics of bipolar and thermally activated delayed fluorescence. By using this material as host of green emitter, (ppy) $)_{2}$ Iracac, high-efficiency and low roll-off phosphorescent organic light-emitting diode (PhOLED) was fabricated with the maximum current efficiency of $56.12 \mathrm{~cd} \cdot \mathrm{A}^{-1}$, the maximum external quantum efficiency of $15.70 \%$ and the maximum power efficiency of $71.3 \mathrm{~lm} \bullet \mathrm{W}^{-1}$. These make DphAn-5PhIdCz a promising host for high performance PhOLED displays and lighting applications.

Keywords indolocarbazole group; organic light-emitting diodes; host; bipolar; thermally activated delayed fluorescence
\end{abstract}

有机发光二极管 (Organic light-emitting diodes,

OLEDs)具有宽视角、超薄、响应迅速以及柔性显示等

\footnotetext{
* Corresponding authors. E-mail: bwei@shu.edu.cn; yshi@shu.edu.cn

Received July 3, 2018; revised September 3, 2018; published online September 26, 2018.

Project supported by the National Natural Science Foundation of China (Nos. 51725505 and 61775130), the National Key Basic Research and Development Program of China (973 program, No. 2015CB655005) and the Science and Technology Committee of Shanghai City (No. 15590500500).

国家自然科学基金(No. 1725505,61775130)、国家重点基础研究发展计划(973 计划, No. 2015CB655005)和上海市科委(No. 15590500500)资助项目.

$\dagger$ 共同第一作者 (These authors contributed equally to this work).
} 
独特优点 ${ }^{[1 \sim 3]}$, 在手机等小屏幕显示领域已被成功应用, 同时在大尺寸显示和室外照明领域 ${ }^{[4,5]}$ 也展现出一定的 应用潜力. 许多研究致力于提高 OLEDs 的性能, 希望器 件具有高的发光效率, 并展现出低的效率滚降和低功耗 特性.

近年来, 研究者们通过引入新发光材料和优化器件 结构来改善传统 OLED 器件的性能, 并已取得了丰硕的 成果. 对于磷光发射常用的传统主体材料通常是单极性 传输材料, 例如 9,9'-(1,3-苯基)二- $9 H$-咔唑(MCP)、4,4'二(9-咔唑)联苯(CBP)等, 基于它们的 OLED 器件已实现 高于 $20 \%$ 的外量子效率(EQE), 但由于这些化合物的单 重态 $\left(S_{1}\right)$ 和三重态 $\left(T_{1}\right)$ 之间的能隙较大, 且高的 $T_{1}$ 态总 是伴随着更高的 $\mathrm{S}_{1}$ 态, 与相邻功能层能级不匹配, 导致 三线态激子浓度过大而淬灭 ${ }^{[6,7]}$, 对磷光有机电致发光 二极管(PhOLEDs)的效率产生不利影响. Lee 等 $^{[8]}$ 将电子 传输材料 1,3,5-三(1-苯基- $1 H$-苯并咪唑- 2-基)苯(TPBi) 和空穴传输材料 4,4',4"-三(咔唑-9-基)三苯胺(TCTA)混 合作为发光层主体掺杂 $\operatorname{Ir}(\mathrm{ppy})_{3}$ 制备绿色磷光 OLED, 通过拓宽激子复合发光区域、降低激子泄漏来降低器件 效率下降问题, 从而使得 OLED 器件的性能得到明显改 善. $\mathrm{Ma}$ 等 ${ }^{[9]}$ 设计了一种类似于低聚物的主体材料, 通过 分离的化学通道抑制三线态-三线态湮灭和三线态-极 化子淬灭效应. 空穴传输型和电子传输型主体结合可能 导致激态复合物形成, 赋予器件低驱动电压和低效率滚

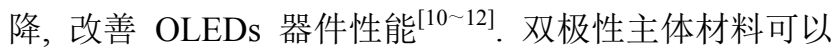
改善载流子迁移率以及发光层中电子和空穴通量的平 衡 $^{[13]}$, Liu 等 ${ }^{[14]}$ 展示了一种具有双极性电荷传输的新型 磷光材料, 其能够承载高效率的深红色 PhOLED; Choi 等 ${ }^{[15]}$ 使用双极性 9-(8-(9H-咔唑-9-基)二苯并 $[\mathrm{b}, \mathrm{d}]$ 噻吩-2基)-9H-吡啶并[2,3- $b$ ] 吲哚材料作主体, 获得了外量子产 率高达 $25.7 \%$ 的蓝色热激发延迟苂光 OLED. Cheng ${ }^{[16]}$ 、 $\mathrm{LiaO}^{[17]}$ 以及 Wang 等 ${ }^{[18]}$ 报道了使用热激发延迟苂光 (TADF)分子作磷光 OLED 的主体材料, 以实现单线态 和三线态能量完全转移到发光体利用, 以及通过激子由 三重态到单重态的有效上转换减少器件效率滚降. Wei 等 ${ }^{[19]}$ 使用具有双极性及热激发延迟苂光(TADF)特性的 11-[3-(4,6-二苯基-1,3,5-三嗪-2-基)苯基]-12,12-二甲基 $-11,12$-二氢狮并 $[2,1-a]$ 咔唑作磷光 OLED 主体材料, 获 得高效率、低滚降的高性能 OLED. OLED 器件性能的 提高是推动其产业化并迅速发展的前提和基础, 高效稳 定的 OLED 器件可以使其在使用中降低功耗, 延长使用 寿命, 提高与传统器件的竞争力.

根据光化学原理, 有机电致磷光器件通过内转换过 程可以同时捕获单重态和三重态激子, 因而理论上可以 达到 $100 \%$ 的内部量子效率. 对于有机电致磷光器件,
通常是将磷光材料作为客体掺杂于主体材料中，或通过 化学键将客体与主体材料相连形成单一分子结构, 将主 客体材料作为发光层, 通过引入合适的电子、空穴注入 和传输层, 在外加电场作用下, 激子辐射衰减发出磷光. 因此, 为了得到性能优异的有机电致磷光器件, 需要设 计合适的主客体材料和优化器件结构. 同时, 除了制作 工艺和结构上的优化外, 根本的解决办法就是从材料方 面着手，只有高效稳定的材料才能在 OLED 的性能上有 所突破. 主体材料通常分为空穴传输型主体材料、电子 传输型主体材料、双极性主体材料. 单极性主体材料存 在弱的载流子迁移率和载流子传输不平衡问题, 形成不 利的窄的激子复合区域. 为了避免这种不利因素，许多 研究将空穴传输型材料与电子传输型材料混合, 达到载 流子平衡的效果, 但这种方法使得制备工艺复杂, 且混 合的主体材料存在相分离问题 ${ }^{[20]}$. 因此, 为了解决上述 问题，需开发具有平衡载流子传输特性的双极性主体材 料.

蒽酮类结构具有吸电子能力, 电子传输能力良好, 玻璃化转移温度和热分解温度高, 具有良好的热稳定性 和化学稳定性 ${ }^{[21]}$; 吲哚并咔唑结构具有给电子能力, 具 有良好的空穴传输能力, 其最高占据分子轨道能级 (5.6 5.8 eV)适合作为主体材料的构建单元, 玻璃化转 移温度和热分解温度高 ${ }^{[22,23]}$. 于此，我们设计合成了一 种新型的双极性主体材料，通过连接基将两基团相连构 成电子供体- $\pi$-电子受体结构, 这种材料具有良好的双 极性特点，能够有利于提高载流子平衡度. 我们从材料 的光学、电化学、热力学方面对材料的光物理性能进行 详细的表征, 通过制备绿色磷光 OLED 器件并测试其发 光性能, 评价该材料作为 OLED 主体材料的性能, 以期 获得优异的 OLED 主体材料. 目标产物合成路线和结构 如 Scheme 1 .

\section{1 结果与讨论}

\section{1 中间体和主体的合成}

中间体材料 10-(4-溴苯基)-10-苯基葱-9-(10H)-酮 (DphAn-Br) 的合成主要来源于文献报道 ${ }^{[21]}$. 第一步在酸 性条件下葱-9,10-二酮与乙二醇进行脱水反应生成中间 体 10H-螺[蒽-9,20-[1,3]二氧戊环]-10-酮(1), 然后加入镁 屑、溴碘苯、苯进行脱水取代反应得到中间体 10,10-二 苯基-10H-螺[葸-9,20-[1,3]二氧戊环](2), 接着中间体 2 与甲酸进行缩铜反应生成中间体 DphAn-Br. 最后 DphAn-Br 与 5-苯基-5,7-二氢吲哚并[2,3-b]咔唑进行偶 联反应生成 DphAn-5PhIdCz (Scheme 1). 结构通过元素 分析、核磁共振氢谱进行确认. 
<smiles>O=C1c2ccccc2C(=O)c2ccccc21</smiles><smiles>COC(=O)OCCO</smiles><smiles>Brc1ccc(C(Br)(Br)Br)cc1</smiles><smiles>CCC1(C)c2ccccc2C2(OCCO2)c2ccccc21</smiles><smiles>OCc1ccc(Br)cc1</smiles><smiles>Brc1ccc(C2(c3ccccc3)c3ccccc3C3(OCCO3)c3ccccc32)cc1</smiles><smiles>O=C1c2ccccc2C(c2ccccc2)(c2ccc(Br)cc2)c2ccccc21</smiles>

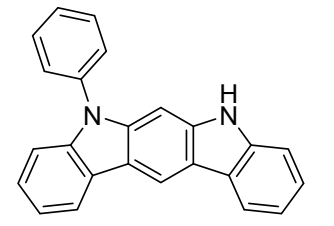

$\mathrm{Pd}_{2}(\mathrm{dba})_{3}, \mathrm{P}(t-\mathrm{Bu})_{3}$ Potassium $t$-butoxide Toluene

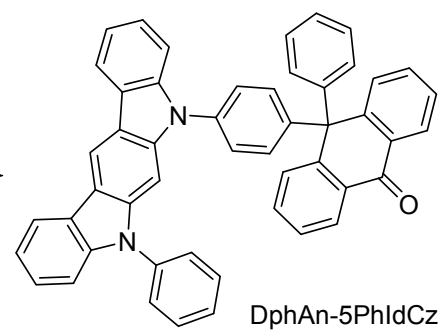

图式 1 目标产物 DphAn-5PhIdCz 的合成路线 Scheme 1 Synthetic route of the target compound DphAn$5 \mathrm{PhIdCz}$

\section{2 主体材料的光物理性能}

为研究合成的主体材料 DphAn-5PhIdCz 的分子结 构和光物理性能之间的关系, 我们利用基于 B3LYP/6$31 \mathrm{G}^{*}$ 的密度泛函理论(DFT)模拟了 DphAn-5PhIdCz 的分 子轨道能级. 图 1 表示材料在最高占据分子轨道 (HOMO) 和最低未占分子轨道(LUMO)状态下的电子云 空间排布. DphAn-5PhIdCz 的 HOMO 轨道的电子云主要 分散在供电子基团二氢苯基吲哚并咔唑 $(\mathrm{PhIdCz})$ 上, 而 其 LUMO 轨道的电子云主要集中在吸电子基团二苯基 葸酮(DphAn)上, 并且通过非共轭部分有效分离 HOMO 和 LUMO 的电子密度. 分离的 HOMO 和 LUMO 轨道有 助于空穴和电子转移平衡, 且引起强电荷转移跃迁, 并 使得其单线态和三线态之间的能量差别很小 ${ }^{[24,25]}$, 因 此，可以降低 OLED 的驱动电压.

利用热重分析法(TGA)和差示扫描量热法(DSC)研 究了 DphAn-5PhIdCz 的热稳定性, 测试结果如图 2. 可 观察到 DphAn-5PhIdCz 的玻璃化转变温度 $\left(T_{\mathrm{g}}\right)$ 为 $144.28{ }^{\circ} \mathrm{C}$ 、分解温度 $\left(T_{\mathrm{d}}\right)$ 为 $401.2{ }^{\circ} \mathrm{C}$, 该温度高于常用 的 CBP 主体材料 $\left(T_{\mathrm{g}}=62{ }^{\circ} \mathrm{C}\right)^{[26]}$. 表明使用 DphAn$5 \mathrm{PhIdCz}$ 得到的固体薄膜具有优异的热稳定性, 有利于 形成具有高形态稳定性的均匀的无定形膜.
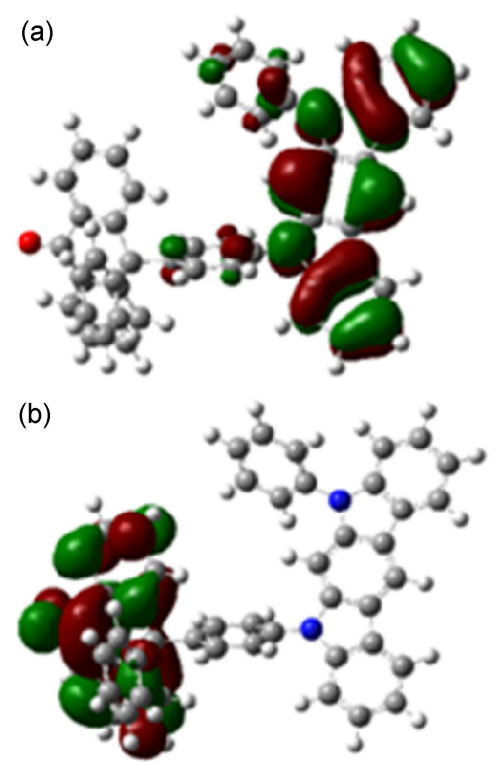

图 1 在 Gaussian 09 DFT B3LYP/6-31G*理论水平中计算的 DphAn-5PhIdCz 的 HOMO (a) 和 LUMO (b)

Figure 1 HOMO (a) and LUMO (b) of DphAn-5PhIdCz as computed in Gaussian 09 DFT B3LYP/6-31G* level of theory
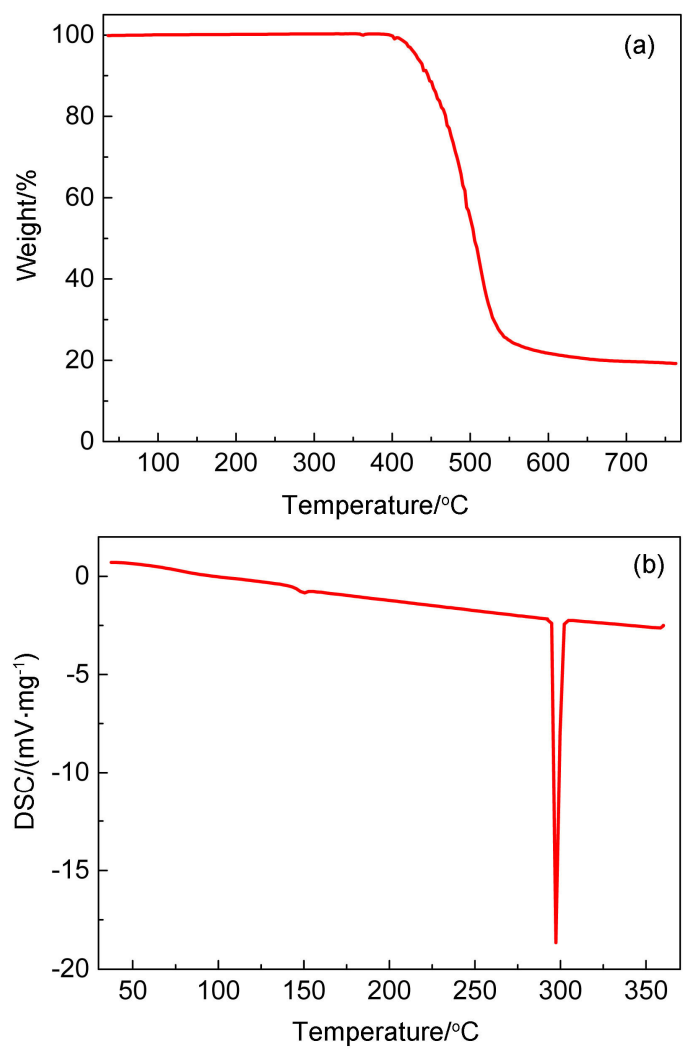

图 2 DphAn-5PhIdCz 的(a)热重分析曲线和(b)差分扫描量热 曲线

Figure 2 (a) Thermal gravimetric analysis curve and (b) differential scanning calorimeter curve of DphAn-5PhIdCz

同时使用循环伏安法测定 DphAn-5PhIdCz 在二氯 甲烷 $\left(\mathrm{CH}_{2} \mathrm{Cl}_{2}\right)$ 溶液中的电化学性质(图 3). 其中 DphAn- 
$5 \mathrm{PhIdCz}$ 的氧化曲线来源于供电子的二氢苯基吲哚并咔 唑基团被氧化; 还原曲线是由于吸电子的二苯基葱酮被 还原. 从图 3 中还可以看出其氧化还原是可逆的, 表明 其具有良好的电化学稳定性 ${ }^{[27]}$.
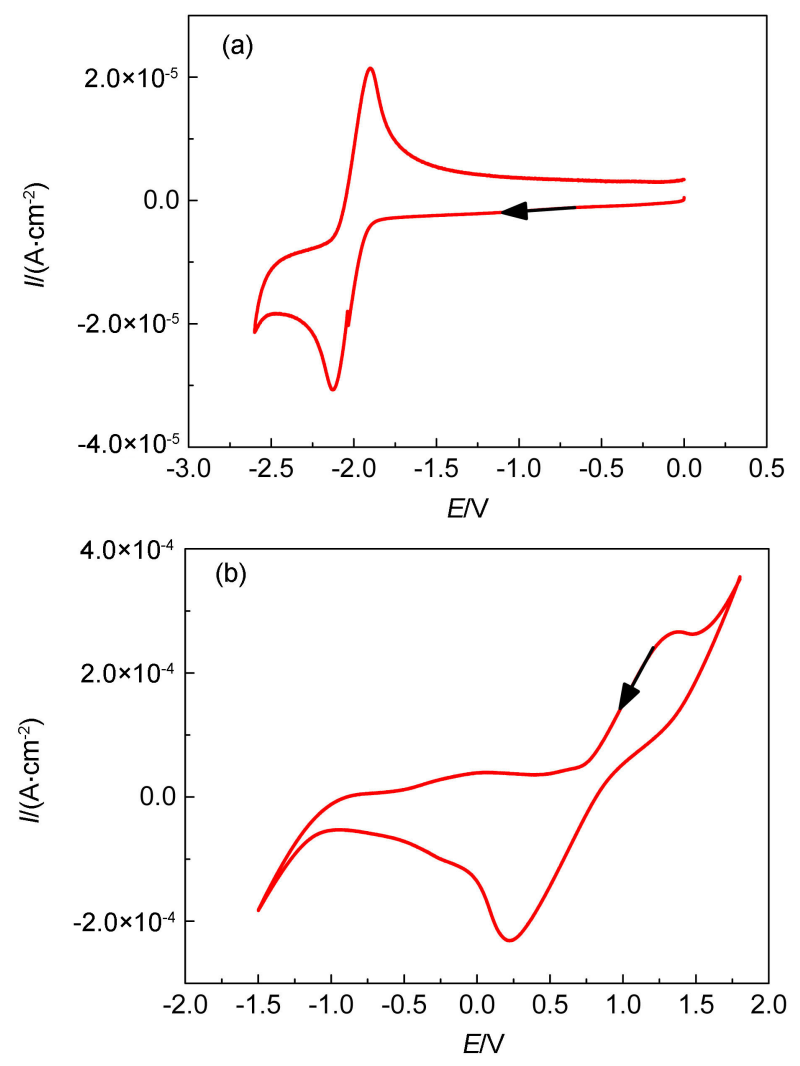

图 3 DphAn-5PhIdCz 的循环伏安曲线

Figure 3 Cyclic voltammetry (CV) curves of DphAn-5PhIdCz Reduction potential; (b) oxidation potential

图 4(a)显示了 DphAn-5PhIdCz 的紫外-可见吸收 $(\mathrm{Abs})$ 和光致发光(PL)发射光谱. 从紫外-可见吸收光谱 可以看到, 化合物 DphAn-5PhIdCz 在小于 $315 \mathrm{~nm}$ 区域 的吸收为 $\pi-\pi *$ 跃迁, 315 和 $370 \mathrm{~nm}$ 附近的吸收为 $n-\pi *$ 跃 迁. 在固体薄膜中观察到 DphAn-5PhIdCz 的最大 PL 发 射波长为 $500 \mathrm{~nm}$; 室温下, 在甲苯溶液 $\left(10^{-5} \mathrm{~mol} / \mathrm{L}\right)$ 中 观察到 DphAn-5PhIdCz 的最大 PL 发射波长为 $386 \mathrm{~nm}$. 对比发现, 薄膜状态相对于溶液中的 PL 光谱发生红移, 主要原因是薄膜状态下, 分子的堆积要比溶液状态下严 重, 分子间作用力增强 ${ }^{[28]}$. 低温下 $(77 \mathrm{~K}$, 液氮)在甲苯 溶液中测量 DphAn-5PhIdCz 的磷光光谱, 从磷光光谱的
最高能量振动峰得到两者的三线态能级为 $2.70 \mathrm{eV}$. DphAn-5PhIdCz 的 PL 光谱和(ppy) ${ }_{2}$ Iracac 的紫外-可见 吸收光谱具有部分重叠, 说明主客体直接搭配有利于能 量转移. 表 1 中总结了 DphAn-5PhIdCz 的光物理性能相 关数据.
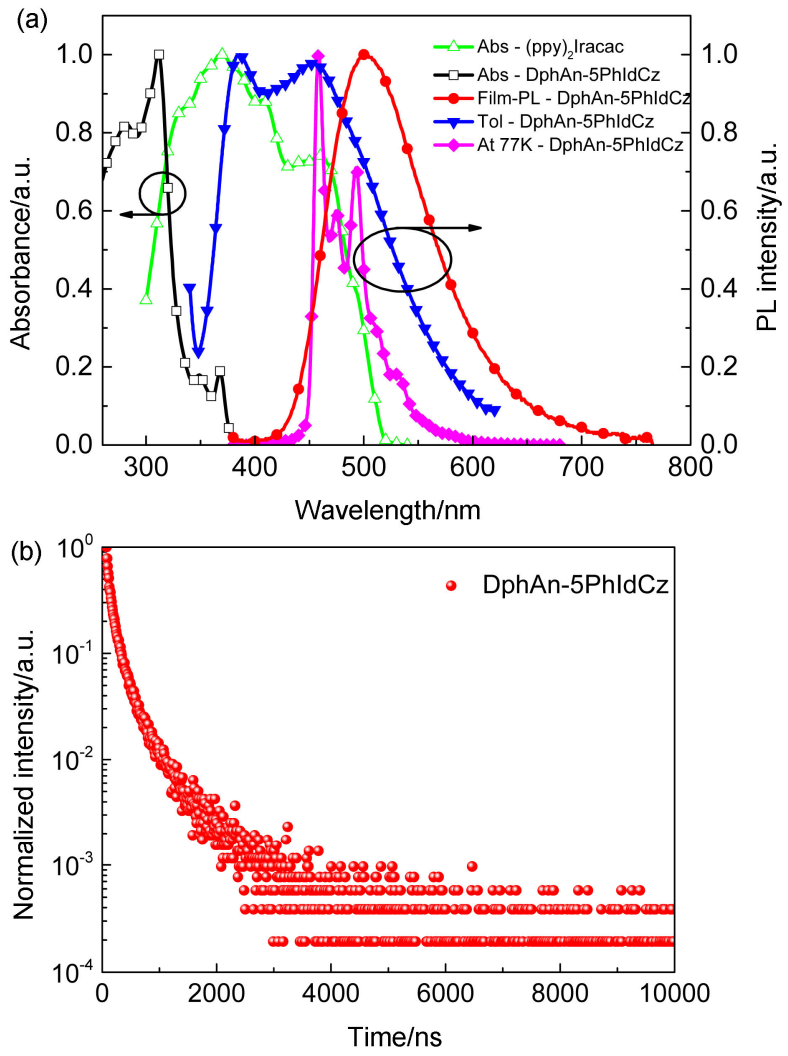

图 4 (a)室温下 $(\text { ppy })_{2} \operatorname{Iracac}$ 吸收光谱和 DphAn-5PhIdCz 的紫 外-可见吸收光谱、PL 光谱、低温磷光光谱; (b)室温下 DphAn$5 \mathrm{PhIdCz}$ 固体薄膜的瞬态荧光衰减曲线

Figure 4 (a) Absorption spectra of (ppy) $)_{2}$ Iracac and Absorption, PL and phosphorescent spectra $(77 \mathrm{~K})$ of DphAn-5PhIdCz; (b) the transient decay curve of $\mathrm{DphAn}-5 \mathrm{PhIdCz}$ film at room temperature

为了研究该主体材料的 TADF 特性, 对其瞬态苂光 寿命进行测试，并且测试了材料的苂光量子产率. 从图 4(b)中可以看出, DphAn-5PhIdCz 瞬态衰减曲线可以分 解为两个成分: 瞬态成分和延迟成分. 通过二阶指数衰 减模型，我们估算出 DphAn-5PhIdCz 的瞬态苂光寿命和 延迟苂光寿命分别为 $18.65 \mathrm{~ns}$ 和 $2.69 \mu \mathrm{s}$. 因此, 通过 DphAn-5PhIdCz 材料的瞬态荧光光谱可以确认该材料

表 1 DphAn-5PhIdCz 的光物理性能相关数据总结

Table 1 Summary of the physical properties of DphAn-5PhIdCz

\begin{tabular}{|c|c|c|c|c|c|c|}
\hline$T_{\mathrm{g}}, T_{\mathrm{m}}, T_{\mathrm{d}} /{ }^{\circ} \mathrm{C}$ & $\mathrm{HOMO} \mathrm{LUMO}^{a} / \mathrm{eV}$ & $T_{1}^{b} / \mathrm{eV}$ & $\Delta E_{\mathrm{ST}}^{c} / \mathrm{eV}$ & $\lambda_{\mathrm{abs}}^{d} / \mathrm{nm}$ & $\lambda_{\mathrm{em}} / \mathrm{nm}$ & $\eta_{\mathrm{PL}} / \%$ \\
\hline DphAn-5PhIdCz 144.28, 295.48, 401.2 & $-5.16,-2.35$ & 2.7 & 0.88 & 312 & $500^{e} / 386^{d} 18.65 \mathrm{~ns} / 2.69 \mu \mathrm{s}$ & 15.48 \\
\hline
\end{tabular}


的延迟苂光来源于 $T_{1}$ 通过反隙间穿越转换为 $S_{1}$ 发光, 表明其具有 TADF 特性. 另外, DphAn-5PhIdCz 薄膜的 荧光量子产率为 $55.48 \%$, 说明 DphAn-5PhIdCz 材料具 有良好的发光特性, 期待可以作为绿色磷光器件发光层 的主体材料.

\section{3 基于 DphAn-5PhldCz 主体的磷光 OLED 的光电 性能}

为了评估和比较 DphAn-5PhIdCz 作为绿色磷光的 主体对器件性能的影响, 我们制备了绿色磷光 OLED 器 件. 器件结构为 ITO/HAT-CN $(10 \mathrm{~nm}) / \mathrm{NPB}(50 \mathrm{~nm}) /$ TAPC $(60 \mathrm{~nm}) /$ Host:12\% (ppy) $)_{2}$ Iracac $(40 \mathrm{~nm}) / \mathrm{TPBi}$ : TmPyPb $(1: 1 \mathrm{~mol} / \mathrm{mol}, 35 \mathrm{~nm}) / \mathrm{Liq}(1 \mathrm{~nm}) / \mathrm{Al}(80 \mathrm{~nm})$. ITO 作为阳极, HAT-CN 作为空穴注入层, NPB 作为空穴 传输层, TAPC 作为电子阻挡层. TPBi 和 $\mathrm{TmPyPb}$ 作为电 子传输层, 降低电子传输能垒; 同时作为阻挡层将激子 限制在发光层, 避免能量流失. 发光层以 DphAn$5 \mathrm{PhIdCz}$ 作为主体材料, 掺杂 $12 \%$ (ppy) I $_{2}$ Iracac 作为客体 材料, 利用主客体之间的能量转移, 提高能量利用率, 获得高性能器件. 此外, 选择通常用于绿色磷光器件的 主体材料 CBP 作主体, 并制备对比器件, 器件结构与基 于 DphAn-5PhIdCz 主体的器件结构相同. 通过使用不同 的主体材料, 研究其对于器件性能的影响.

图 5(a)为器件的电流密度-电压-亮度特征曲线, 结 合器件性能参数(表 2 )可知, 在 $1 \mathrm{~cd} \cdot \mathrm{m}^{-2}$ 的亮度下, 基于 CBP 的参照器件的启亮电压高达 $3.1 \mathrm{~V}$, 而基于 DphAn-5PhIdCz 的绿光器件只有 $2.1 \mathrm{~V}$ 的起亮电压. 另 外, $5000 \mathrm{~cd} \cdot \mathrm{m}^{-2}$ 的亮度情况下, 基于 DphAn-5PhIdCz 的 驱动电压是 $4.05 \mathrm{~V}$, 而基于 $\mathrm{CBP}$ 的驱动电压为 $6.27 \mathrm{~V}$. 这表明基于 DphAn-5PhIdCz 的器件能耗大幅度降低. 图 5(b) 为器件的外量子效率-亮度-功率效率特征曲线, 结 合表 2 可以看出, 器件具有较高的效率, 基于 DphAn$5 \mathrm{PhIdCz}$ 主体的器件的最大电流效率达到 $56.12 \mathrm{~cd} \cdot \mathrm{A}^{-1}$, 最大外量子效率 $15.70 \%$, 最大功率效率 $71.3 \mathrm{~lm} \cdot \mathrm{W}^{-1}$, 即使在 $5000 \mathrm{~cd} \cdot \mathrm{m}^{-2}$ 高亮度下, 器件外量子效率和功率 效率仍然高达 $13.3 \%$ 和 $35.85 \mathrm{~lm} \cdot \mathrm{W}^{-1}$, 对比基于 $\mathrm{CBP}$ 的 参照器件, 基于 DphAn-5PhIdCz 的器件在高亮度下性能 比较稳定, 效率滚降得到抑制. 从器件的电致发光光谱 (EL)来看, 所有的 OLED 器件都发射绿光, 基于 CBP 和
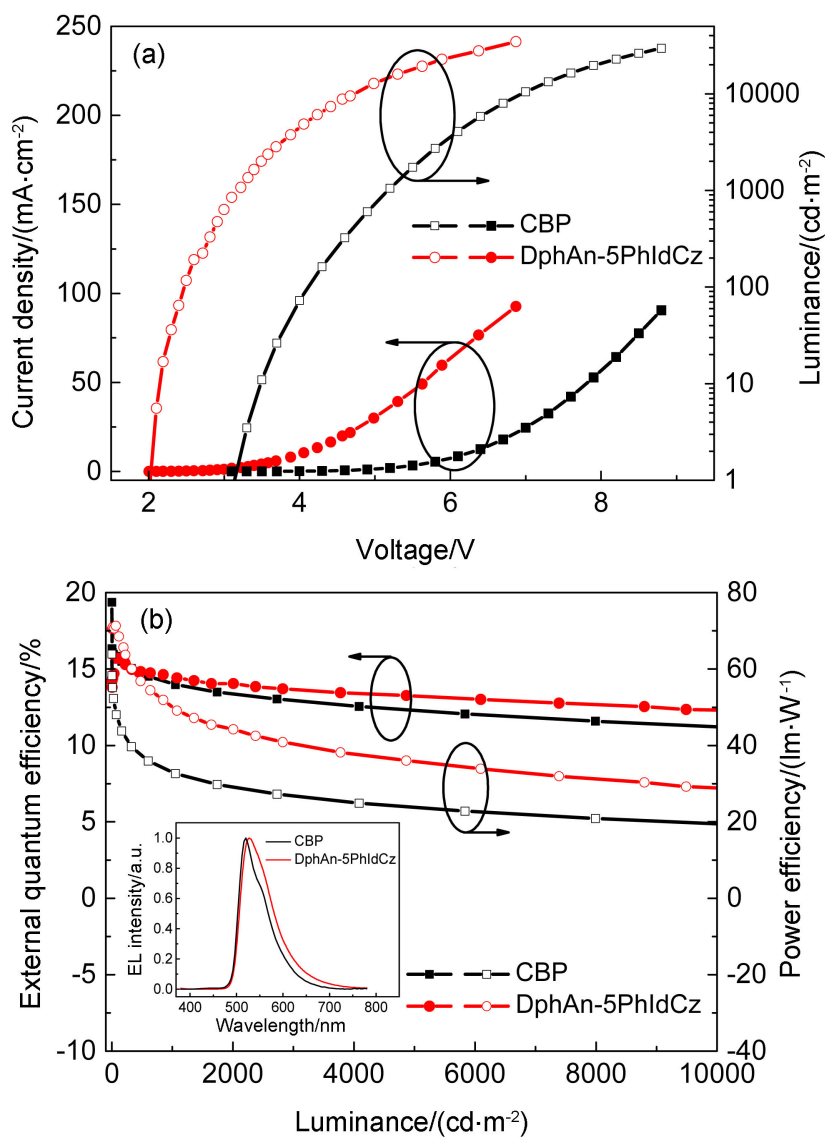

图 5 (a)器件的电流密度-电压-亮度曲线和(b)外量子效率-亮 度一功率效率曲线

Figure 5 (a) Current density-voltage-luminance curves and (b) external quantum efficiency-luminance-power efficiency curves of devices

Inset: the normalized EL spectra

DphAn-5PhIdCz 主体的器件发射峰值分别位于 520 和 $527 \mathrm{~nm}$, 如图 5(b)插图, 但基于 CBP 的器件的 EL 光谱 出现侧峰, 是由于 $\mathrm{CBP}$ 分子的空间平面构型导致, 而 DphAn-5PhIdCz 分子具有空间扭曲结构, 降低了发光分 子的边带(side-band)的发射, 未导致肩峰的存在 ${ }^{[29]}$.

为了进一步调查该材料的双极性特对起亮电压和 效率的影响, 我们制备了单载流子器件, 其中单空穴器 件的结构: ITO/HAT-CN $(10 \mathrm{~nm}) / \mathrm{TAPC}(20 \mathrm{~nm}) / \mathrm{CBP}$ or DphAn-5PhIdCz (20 nm)/TAPC (20 nm)/HAT-CN (10 $\mathrm{nm}) / \mathrm{Al}(100 \mathrm{~nm})$, 单电子器件的结构: ITO/Liq $(1 \mathrm{~nm}) /$ TPBi (30 nm)/CBP or DphAn-5PhIdCz (20 nm)/TPBi (30

表 2 基于 CBP 和 DphAn-5PhIdCz 的磷光 OLEDs 器件性能参数总结

Table 2 Summary on the performance of the PhOLEDs based on CBP and DphAn-5PhIdCz hosts

\begin{tabular}{cccccccc}
\hline Device & $V_{\mathrm{on}}{ }^{a} / \mathrm{V}$ & $\mathrm{CE}_{\max }{ }^{b} /\left(\mathrm{cd} \bullet \mathrm{A}^{-1}\right)$ & $\mathrm{PE}_{\max }{ }^{c} /\left(\mathrm{lm} \bullet \mathrm{W}^{-1}\right)$ & $\mathrm{EQE}_{\max }{ }^{d} / \%$ & $\mathrm{EQE}^{e} / \%$ & $\mathrm{PE}^{e} /\left(1 \mathrm{~m} \bullet \mathrm{W}^{-1}\right)$ & $\mathrm{CIE}(x, y)$ \\
\hline $\mathrm{CBP}$ & 3.1 & 63.1 & 63.90 & 19.37 & $15.70 / 14.10 / 12.10$ & $46.78 / 33.12 / 23.81$ & $(0.32,0.63)$ \\
DphAn-5PhIdCz & 2.1 & 54.5 & 71.30 & 15.70 & $15.69 / 14.51 / 13.30$ & $69.32 / 50.16 / 35.85$ & $(0.35,0.61)$ \\
\hline
\end{tabular}

${ }^{a}$ Turn-on voltages estimated at the luminance of $1 \mathrm{~cd} \bullet \mathrm{m}^{-2} .{ }^{b}$ Maximum current efficiency. ${ }^{c}$ Maximum power efficiency. ${ }^{d}$ Maximum external quantum efficiency. ${ }^{e}$ The efficiencies at the luminance of 100,1000 and $5000 \mathrm{~cd} \cdot \mathrm{m}^{-2}$, respectively. 

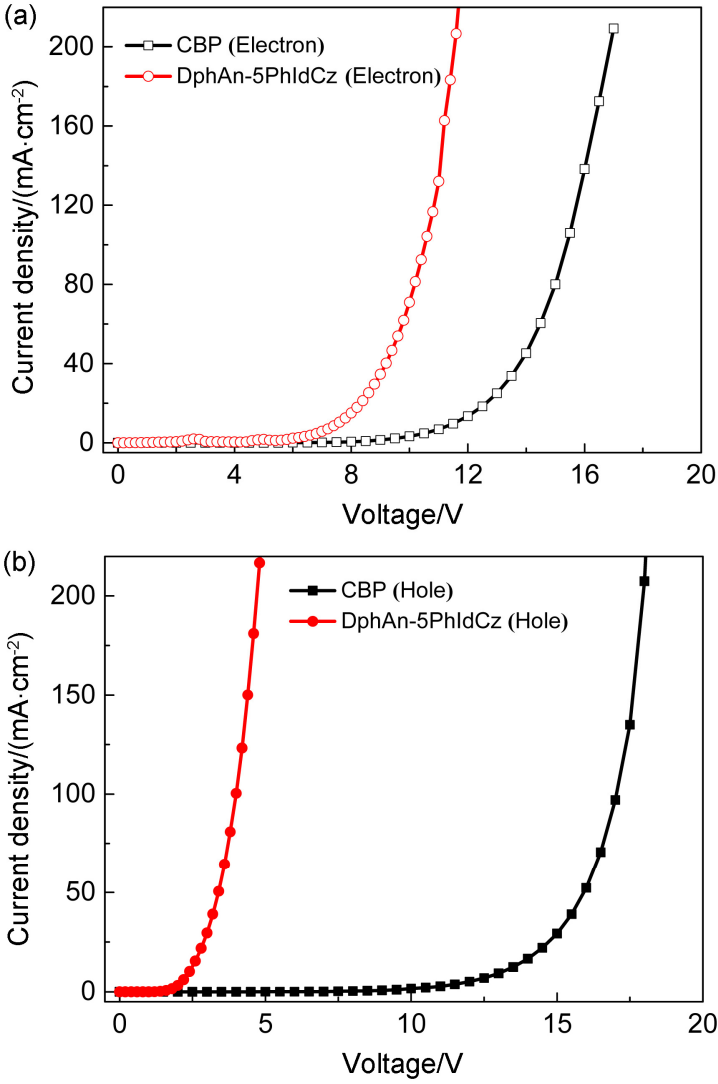

图 6 单载流子器件的电流密度-电压特征曲线

Figure 6 Current density-voltage characteristics of cells (a) Electron-only cells; (b) hole-only cells

$\mathrm{nm}) / \mathrm{Liq}(1 \mathrm{~nm}) / \mathrm{Al}(100 \mathrm{~nm})$. 由单载流子器件的电流密 度-电压特征曲线(图 6)可知, 基于 DphAn-5PhIdCz 主体 的器件中的电子和空穴向发光层的注入能力较好且电 子和空穴的传输速率相当, 这有利于降低器件的驱动电 压和器件能耗. 另一方面, 器件具有较高的效率是由于 新材料 DphAn-5PhIdCz 具有 TADF 效应, 三重态激子能 够通过反隙间穿越转换到单重态, 降低了三重态激子的 浓度，从而降低了三线态激子的浓度淬灭效应，提高了 激子利用率, 使得器件有较高的性能.

\section{2 结论}

设计并合成了一种新型的绿光主体材料 DphAn$5 \mathrm{PhIdCz}$ ，并通过元素分析、核磁氢谱对其结构进行了表 征, 研究了其光物理性能, 主体材料 DphAn-5PhIdCz 显 示出良好的发光性能和热稳定性. 通过将 DphAn$5 \mathrm{PhIdCz}$ 作为绿色发光体(ppy) I $_{2}$ racac 的主体, 成功制备 了高效低滚降的磷光 OLED 器件, 其最大发光效率达到 $56.12 \mathrm{~cd} \cdot \mathrm{A}^{-1}$, 最大外量子效率 $15.70 \%$, 最大功率效率 $71.3 \mathrm{~lm} \cdot \mathrm{W}^{-1}$, 因此在提高磷光 OLEDs 综合性能方面是 非常有效的, 且优越的性能使 DphAn-5PhIdCz 主体有望
应用于高性能 OLED 显示器和照明.

\section{3 实验部分}

\section{1 仪器与试剂}

德国 Elementar 公司 Vario MACRO cube 型元素分 析仪. ${ }^{1} \mathrm{H}$ NMR 采用 BRUKER AVANCE-500 核磁共振波 谱仪测定, TMS 为内标, $\mathrm{CDCl}_{3}$ 为溶剂. 材料的热稳定性 通过热重分析法(TGA)和差分扫描量热法(DSC)来进行 分析. 氧化还原电位用循环伏安法测定, 用 $0.1 \mathrm{~mol} \cdot \mathrm{L}^{-1}$ 六氟磷酸四正丁基胺 $\left(n-\mathrm{Bu}_{4} \mathrm{NPF}_{6}\right)$ 作为支持电解质，铂 作为工作电极, 铂丝作为辅助电极, 二茂铁/二茂铁盐标 定的银线作为参比电极, 使用恒电位/恒电流仪 283 型 (普林斯顿应用研究所) 电化学工作站测量 DphAn$5 \mathrm{PhIdCz}\left(\mathrm{CH}_{2} \mathrm{Cl}_{2}\right.$ 溶液, 浓度 $\left.2 \times 10^{-3} \mathrm{~mol} \cdot \mathrm{L}^{-1}\right)$ 的还原/氧 化电位, 扫描速率为 $100 \mathrm{mV} \cdot \mathrm{s}^{-1}$. 吸收光谱使用紫外可见分光光度计测试. 发射光谱、瞬态荧光寿命、荧光 量子产率使用 FLSP920 荧光光谱仪测量. OLED 器件电 致发光光谱和电流密度-电压-亮度特性使用苏州弗士 达科学仪器有限公司的 FS-1000GA 测试系统测量. 实 验所用试剂均为国产分析纯.

\subsection{DphAn-5PhldCz 的合成}

按照文献报道 ${ }^{[21]}$ 的方法合成了中间体 10-(4-溴苯 基)-10-苯基葱-9-(10H)-酮(DphAn-Br).

在通氮气保护下, 往三口烧瓶中依次加入 $3.32 \mathrm{~g}$ (10 mmol) 5-苯基-5,7-二氢吲哚并[2,3- $b$ ]咔唑、4.68 g DphAn-Br (11 mmol)、3.37 g (30 mmol)叔丁醇钾, $0.02 \mathrm{~g}$ $(0.22 \mathrm{mmol})$ 三二亚苄基丙酮二钯和 $100 \mathrm{~mL}$ 甲苯, 加热 至回流，保温反应 $12 \mathrm{~h}$ 后冷却至室温，进行过滤，用二 氯甲烷淋洗 2 次. 减压蒸馏除去有机溶剂, 用 $V$ (石油 醚) $: V$ (二氯甲烷 $)=5: 1$ 的混合溶剂进行硅胶柱层析, 得到 DphAn-5PhIdCz 淡黄色固体粉末 $5.59 \mathrm{~g}$, 产率 82.6\%, HPLC 纯度 $99.3 \% .{ }^{1} \mathrm{H}$ NMR $\left(500 \mathrm{MHz}, \mathrm{CDCl}_{3}\right) \delta$ : $8.84 \sim 87.7(\mathrm{~m}, 1 \mathrm{H}), 8.35(\mathrm{dd}, J=7.7,1.5 \mathrm{~Hz}, 2 \mathrm{H}), 8.24$ (dd, $J=7.2,6.2 \mathrm{~Hz}, 2 \mathrm{H}), 7.61 \sim 7.58(\mathrm{~m}, 4 \mathrm{H}), 7.57 \sim 7.47$ (m, 5H), $7.46 \sim 7.42(\mathrm{~m}, 2 \mathrm{H}), 7.41 \sim 7.35(\mathrm{~m}, 4 \mathrm{H}), 7.34 \sim$ $7.27(\mathrm{~m}, 5 \mathrm{H}), 7.26 \sim 7.19(\mathrm{~m}, 5 \mathrm{H}), 7.11 \sim 7.06(\mathrm{~m}, 2 \mathrm{H})$, $5.30(\mathrm{~s}, 1 \mathrm{H})$. Anal. calcd for $\mathrm{C}_{50} \mathrm{H}_{32} \mathrm{~N}_{2} \mathrm{O}: \mathrm{C} 88.73, \mathrm{H} 4.77$, N 4.14; found C 88.875, H 4.710, N 4.199.

\section{3 吸收光谱、发射光谱、瞬态荧光寿命和荧光量子 产率的测试}

用甲苯作溶剂, 分别测定了目标产物溶液状态下的 PL 光谱; 低温下 $(77 \mathrm{~K}$, 液氮) 测试了磷光光谱, 同时也 测定了固体薄膜的吸收光谱、发射光谱、瞬态苂光寿命 和苂光量子产率. 


\subsection{OLED 器件的制备和发光性能}

OLED 器件采用真空热蒸镀的方法制备, 除金属 $\mathrm{Al}$ 电极蒸发速率为 $0.50 \mathrm{~nm} / \mathrm{s}$ 外, 其余材料蒸发速率均为 $0.06 \mathrm{~nm} / \mathrm{s}$. 材料的蒸发速度及厚度用石英晶振测量, 器 件发光面积为 $4 \mathrm{~mm}^{2}$. 以 DphAn-5PhIdCz 作为发光主体 制备了 OLED 器件, 并测试了相关发光性能.

辅助材料(Supporting Information) OLED 器件的能级 结构图、新材料 DphAn-5PhIdC 的 ${ }^{1} \mathrm{H}$ NMR 图. 这些材 料可以免费从本刊网站(http://sioc-journal.cn/)上下载.

\section{References}

[1] Wang, Z. B.; Helander, M. G.; Qiu, J.; Puzzo, D. P.; Greiner, M. T.; Hudson, Z. M.; Wang, S.; Liu, Z. W.; Lu, Z. H. Nat. Photon. 2011, 5,753 .

[2] Chen, M.; Zhang, L.; Lin, H.; Li, Q.; Yu, J. S. J. Optoelectron $\bullet$ Laser 2012, 23, 1063 (in Chinese). (陈珢, 张砧, 林慧, 李青, 于军胜, 光电子・激光, 2012, 23, 1063.)

[3] Ding, L.; Zhang, F. H.; Li, Y. F.; Liang, T. J.; Zhang, J. J. Optoelectron $\bullet$ Laser 2011, 22, 1615 (in Chinese). (丁否, 张方辉, 李艳飞, 梁田静, 张静, 光电子・激光, 2011, 22, 1615.)

[4] Nakayama, T.; Hiyama, K.; Furukawa, K.; Ohtani, H. Sid Symp. Dig. Tech. Pap. 2007, 38, 1018.

[5] Zhou, T. X.; Tan, N.; Brown, J. J.; Shtein, M..; Forrest, S. R. Appl. Phys. Lett. 2005, 86, 3033.

[6] Wang, Q.; Oswald, I. W. H.; Yang, X. L.; Zhou, G. J.; Jia, H. P.; Qiao, Q. Q.; Hoshikawa-Halbert, J.; Gnade, B. E. Adv. Electron. Mater. 2015, 1, 322.

[7] Yu, D. H.; Zhao, F. C.; Han, C. M.; Xu, H.; Li, J.; Zhang, Z.; Deng, Z. P.; Ma, D. G.; Yan, P. F. Adv. Mater. 2012, 24, 509.

[8] Kim, S. H.; Jang, J.; Yook, K. S.; Lee, J. Y. Appl. Phys. Lett. 2008, 92, 023513 .

[9] Han, C. H.; Zhu, L. P.; Li, J.; Zhao, F. C.; Zhang, Z.; Xu, H.; Deng, Z. P.; Ma, D. G.; Yan, P. F. Adv. Mater. 2014, 26, 7070.

[10] Park, Y. S.; Lee, S.; Kim, K. H.; Kim, S. Y.; Lee, J. H.; Kim, J. J. Adv. Funct. Mater. 2013, 23, 4914.
[11] Shin, H.; Lee, S.; Kim, K. H.; Moon, C. K.; Yoo, S. J.; Lee, J. H.; Kim, J. J. Adv. Mater. 2014, 26, 4730.

[12] Lee, J. H.; Cheng, S. H.; Yoo, S. J.; Shin, H.; Chang, J. H.; Wu, C. I.; Wong, K. T.; Kim, J. J. Adv. Funct. Mater. 2015, 25, 361.

[13] Tao, Y. T.; Wang, Q.; Yang, C. L.; Wang, Q.; Zhang, Z. Q.; Zou, T. T.; Qin, J. G.; Ma, D. G. Angew. Chem. 2008, 120, 8224.

[14] Feng, Y. S.; Ping Li, P.; Zhuang, X. M.; Ye, K. Q.; Peng, T.; Liu, Y.; Wang, Y. Chem. Commun. 2015, 51, 12544.

[15] Kang, J. S.; Hong, T. R.; Kim, H. J.; Son, Y. H.; Lampande, R.; Kang, B. Y.; Lee, C.; Bin, J. K.; Lee, B. S.; Yang, J. H.; Kim, J.; Park, S.; Cho, M. J.; Kwon, J. H.; Choi, D. H. J. Mater. Chem. 2016, 4, 4512 .

[16] Lin, C. C.; Huang, M. J.; Chiu, M. J.; Huang, M. P.; Chang, C. C.; Liao, C. Y.; Chiang, K. M.; Shiau, Y. J.; Chou, T. Y.; Chu, L. K.; Lin, H. W.; Cheng, C. H. Chem. Mater. 2017, 29, 1527.

[17] Liu, Y. Y.; Liang, F.; Yuan, Y.; Cui, L. S.; Jiang, Z. Q.; Liao, L. S. Chem. Commun. 2016, 52, 8149.

[18] Wang, H.; Meng, L. Q.; Shen, X. X.; Wei, X. F.; Zheng, X. L.; Lv, X. P.; Yi, Y. P.; Wang, Y.; Wang, P. F. Adv. Mater. 2015, 27, 4041.

[19] Guo, K. P.; Wang, H. D.; Wang, Z. X.; Si, C. F.; Peng, C. Y.; Wei, B. Chem. Sci. 2017, 8, 1259.

[20] Zhang, H. H.; Wu, H. Imag. Sci. Photochem. 2015, 33, 183 (in Chinese). (张慧慧, 吴昊, 影像科学与光化学, 2015, 33, 183.)

[21] Gao, Z. L.; Liu, Y. R.; Shi, Y.; Wang, Y. X.; Xu, Y.; Xu, Q. CN $106008264,2017$.

[22] Wu, Y.; Li, Y. N.; Gardner, S.; Ong, B. S. J. Am. Chem. Soc. 2004, $127,614$.

[23] Li, Y. N.; Wu, Y. L.; Gardner, S.; Ong, B. S. Adv. Mater. 2005, 17, 849.

[24] Endo, A.; Sato, K.; Yoshimura, K.; Kai, T.; Kawada, A.; Miyazaki, H.; Adachi, C. Appl. Phys. Lett. 2011, 98, 42.

[25] Uoyama, H.; Goushi, K.; Shizu, K.; Nomura, H.; Adachi, C. Nature 2012, 492, 234.

[26] Tsai, M. H.; Hong, Y. H.; Chang, C. H.; Su, H. C.; Wu, C. C.; Mato-liukstyte, A.; Simokaitiene, J.; Grigalevicius, S.; Grazulevicius, J. V.; Hsu, C. P. Adv. Mater. 2007, 19, 862.

[27] Zhang, D. D.; Cai, M. H.; Zhang, Y.G.; Zhang, D. Q.; Duan, L. Mater. Horiz. 2016, 3, 145.

[28] Zeng, Q.; Li, Z.; Dong, Y. Q.; Di, C. A.; Qin, J. G.; Tang, B. Z. Chem. Commun. 2007, (1), 70.

[29] Guo, K. P.; Zhang, J. H.; Xu, T.; Gao, X. C.; Wei, B. J. Disp. Technol. 2014, 10, 642 . 